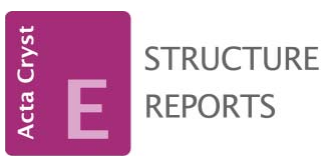

ISSN $1600-5368$

Received 14 November 2014

Accepted 24 November 2014

Edited by $\mathrm{H}$. Stoeckli-Evans, University of

Neuchâtel, Switzerland

† Additional correspondence author, e-mail: rajakannan@unom.ac.in

\section{Crystal structure of 3-amino-1-propylpyridinium bromide}

\author{
P. Venkatesan, ${ }^{\mathrm{a}}$ V. Rajakannan ${ }^{\mathrm{b}} \ddagger$ and S. Thamotharan ${ }^{\mathrm{c} *}$ \\ ${ }^{\mathbf{a}}$ School of Chemistry, Bharathidasan University, Tiruchirappalli 620 024, India, ${ }^{\mathbf{b} C e n t r e}$ of Advanced Study in \\ Crystallography and Biophysics, University of Madras, Chennai 600 025, India, and 'Department of Bioinformatics, \\ School of Chemical and Biotechnology, SASTRA University, Thanjavur 613 401, India. *Correspondence e-mail: \\ thamu@scbt.sastra.edu
}

The title molecular salt, $\mathrm{C}_{8} \mathrm{H}_{13} \mathrm{~N}_{2}{ }^{+} \cdot \mathrm{Br}^{-}$, crystallizes with two independent 3 -aminopyridinium cations and two bromide anions in the asymmetric unit $\left(Z^{\prime}=\right.$ 2 ). In the pyridine ring, the $\mathrm{N}$ atom is alkylated by a propyl group. The dihedral angle between the mean planes of the pyridinium ring and the propyl group is $84.84(2)^{\circ}$ in cation $A$, whereas the corresponding angle is 89.23 (2) $)^{\circ}$ in cation $B$. In the crystal, the anions and cations are linked via $\mathrm{N}-\mathrm{H} \cdots \mathrm{Br}$ and $\mathrm{C}-\mathrm{H} \cdots \mathrm{Br}$ hydrogen bonds, forming chains propagating along [100].

\section{Chemical context}

Aminopyridinium and 1-alkyl-aminopyridinium salts display a wide range of antimicrobial activity (Sundararaman et al., 2013; Ilangovan et al., 2012). They have found many applications such as surfactants (Gama et al., 1981), ionic liquids (Muldoon et al., 2010; Petkovic et al., 2011), liquid-crystal display mediums (Ezaki \& Kokeguchi, 2006), ionic crystals for second-order non-linear optics (Anwar et al., 2001), phasetransfer catalysts in organic transformations (Kupetis et al., 2002) and additives for protein refolding processes (Yamamoto et al., 2011). In addition, the amino group in the pyridinium ring participates through hydrogen bonds with wool proteins (Zhao \& Sun, 2007; Calas et al., 2007).

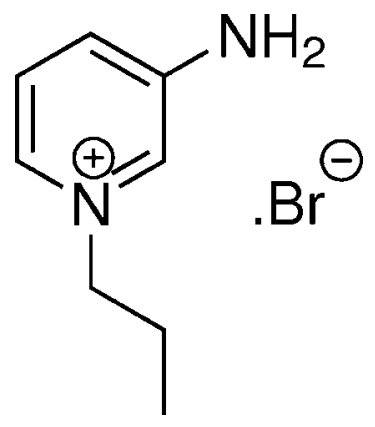

\section{Structural commentary}

The asymmetric unit of the title salt, consists of two 3-aminopyrdinium cations and two bromide anions, as shown in Fig. 1. The geometrical parameters of the cation moiety are comparable with those of a related structure, 3-amino-1-(4nitrobenzyl)pyridinium bromide (Sundar et al., 2006). The molecular structure of the two cations are very similar with weighted and unit-weight r.m.s. fits of 0.089 and $0.081 \AA$, respectively, for ten fitted atoms (Fig. 2). The dihedral angle 


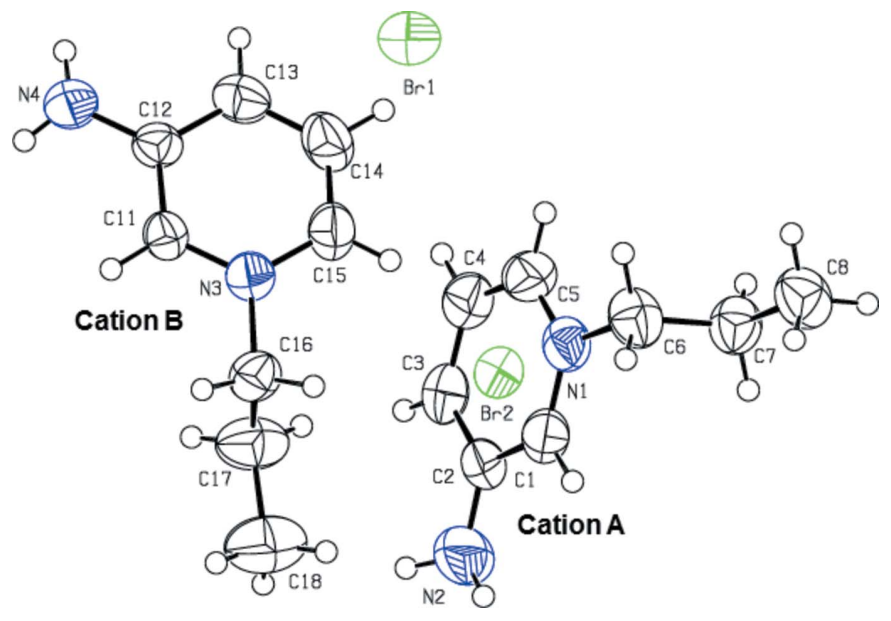

Figure 1

The molecular structure of the title salt, showing the atom labelling. Displacement ellipsoids are drawn at the $50 \%$ probability level.

between the mean planes of the pyridinium ring (N2/C1-C5) and the propyl group (N1/C6-C8) is $84.84(2)^{\circ}$ in cation $A$, whereas the corresponding angle is $89.23(2)^{\circ}$ in cation $B$.

\section{Supramolecular features}

The crystal structure of the title salt, is stabilized by a network of intermolecular $\mathrm{N}-\mathrm{H} \cdot \mathrm{Br}$ and $\mathrm{C}-\mathrm{H} \cdots \mathrm{Br}$ hydrogen bonds (Table 1 and Fig. 3). Anion Br2 is involved in five hydrogen bonds as an acceptor while anion $\mathrm{Br} 1$ is involved in only two hydrogen bonds. The dimerization of cation $A$ mediates through two bromide anions with the aid of two $\mathrm{N}-\mathrm{H} \cdots \mathrm{Br}$ and $\mathrm{C}-\mathrm{H} \cdots \mathrm{Br}$ hydrogen bonds. As shown in Fig. 4, these interactions generate an $R_{4}^{2}(12)$ loop. Atom C16 (via $\mathrm{H} 16 A$ ) forms a $\mathrm{C}-\mathrm{H} \cdots \mathrm{Br}^{\mathrm{i}}$ hydrogen bond with bromide anion $\mathrm{Br} 2$

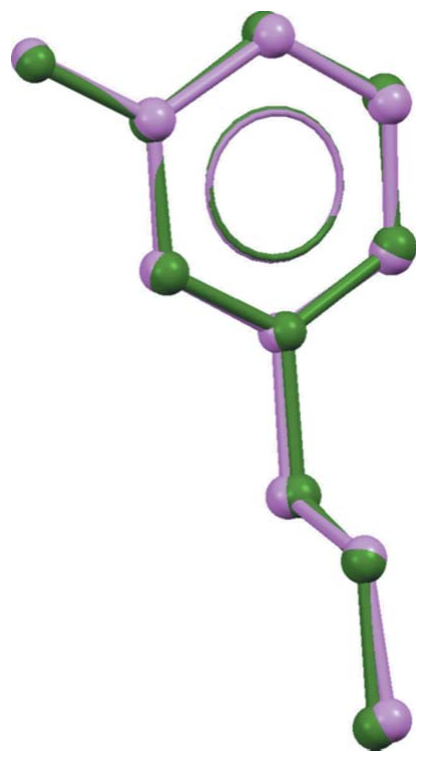

Figure 2

Structural superimposition of the non-H atoms of the pyridinium cations (green: cation $A$; violet: cation $B$ ).
Table 1

Hydrogen-bond geometry $\left(\AA,^{\circ}\right)$.

\begin{tabular}{lllll}
\hline$D-\mathrm{H} \cdots A$ & $D-\mathrm{H}$ & $\mathrm{H} \cdots A$ & $D \cdots A$ & $D-\mathrm{H} \cdots A$ \\
\hline $\mathrm{N} 2-\mathrm{H} 2 A \cdots \mathrm{Br} 2^{\mathrm{i}}$ & $0.90(2)$ & $2.47(2)$ & $3.364(3)$ & $177(4)$ \\
$\mathrm{N} 2-\mathrm{H} 2 B \cdots \mathrm{Br} 2^{\mathrm{ii}}$ & $0.90(2)$ & $2.54(2)$ & $3.419(3)$ & $168(4)$ \\
$\mathrm{N} 4-\mathrm{H} 4 A \cdots \mathrm{Br} 1^{\mathrm{iii}}$ & $0.83(2)$ & $2.58(2)$ & $3.406(3)$ & $172(3)$ \\
$\mathrm{N} 4-\mathrm{H} 4 B \cdots \mathrm{Br} 2^{\text {iv }}$ & $0.87(2)$ & $2.57(2)$ & $3.434(3)$ & $171(3)$ \\
$\mathrm{C} 6-\mathrm{H} 6 A \cdots \mathrm{Br} 2$ & 0.97 & 2.88 & $3.655(4)$ & 138 \\
$\mathrm{C} 6-\mathrm{H} 6 B \cdots \mathrm{Br} 1^{\mathrm{ii}}$ & 0.97 & 2.84 & $3.775(4)$ & 163 \\
$\mathrm{C} 16-\mathrm{H} 16 A \cdots \mathrm{Br} 2^{\mathrm{v}}$ & 0.97 & 2.91 & $3.866(3)$ & 167 \\
\hline
\end{tabular}

Symmetry codes: (i) $x-1, y, z$; (ii) $x-\frac{1}{2},-y+\frac{1}{2},-z+1$; (iii) $-x+\frac{3}{2},-y, z-\frac{1}{2}$; (iv) $x+\frac{1}{2}, y,-z+\frac{1}{2} ;$ (v) $x+1, y, z$.

[symmetry code: (i) $x+1, y, z$ ]. The same $\mathrm{Br} 2$ anion acts as an acceptor for an $\mathrm{N}-\mathrm{H} \cdots \mathrm{Br}$ hydrogen bond with atom $\mathrm{N} 4$ of cation $B$. These interactions form a chain which runs parallel to the $a$ axis (Fig. 5).

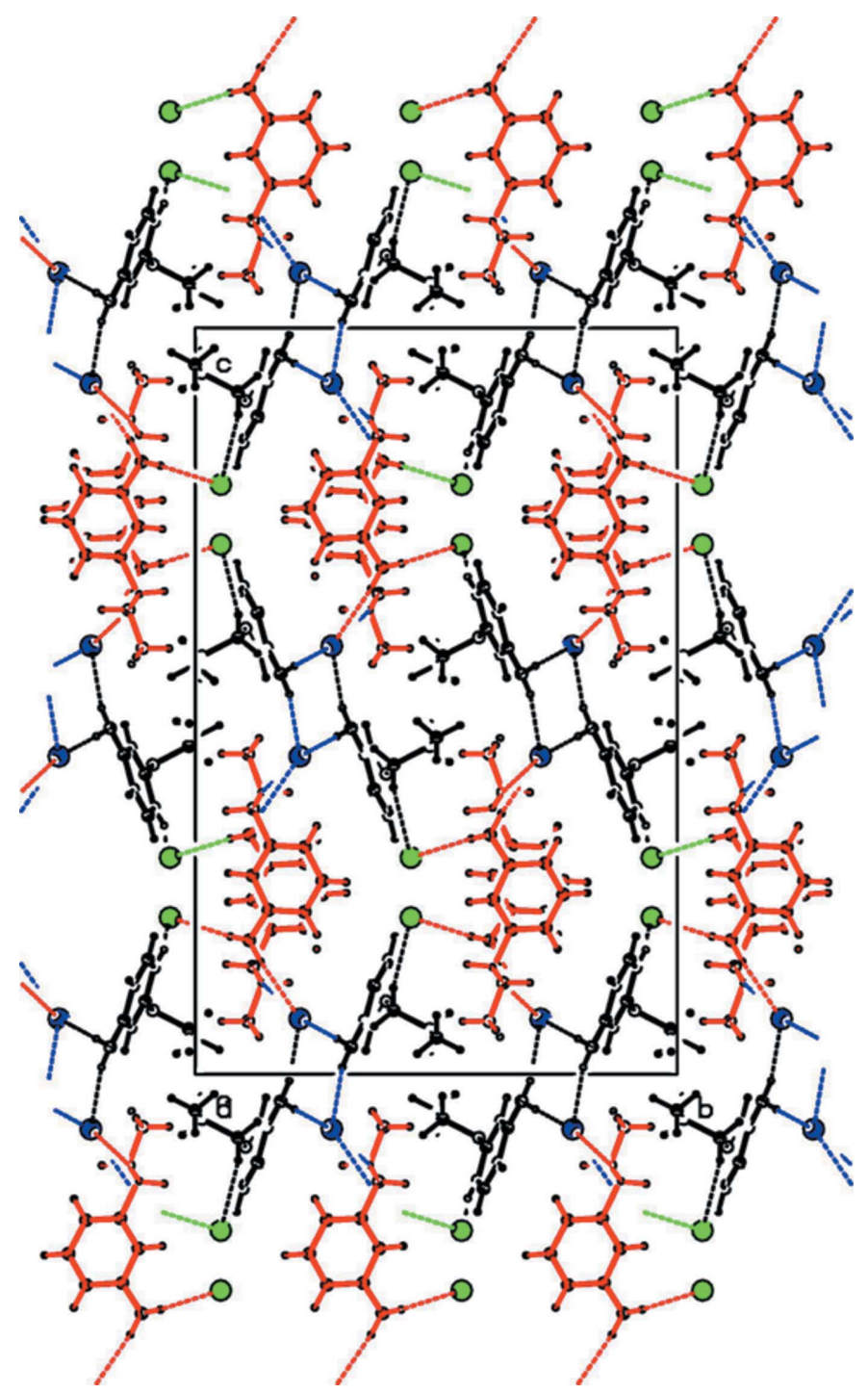

Figure 3

The crystal packing of the title salt projected onto the $b c$ plane. The $\mathrm{N}-$ $\mathrm{H} \cdots \mathrm{Br}$ and $\mathrm{C}-\mathrm{H} \cdots \mathrm{Br}$ hydrogen bonds are shown as dashed lines (see Table 1 for details). 


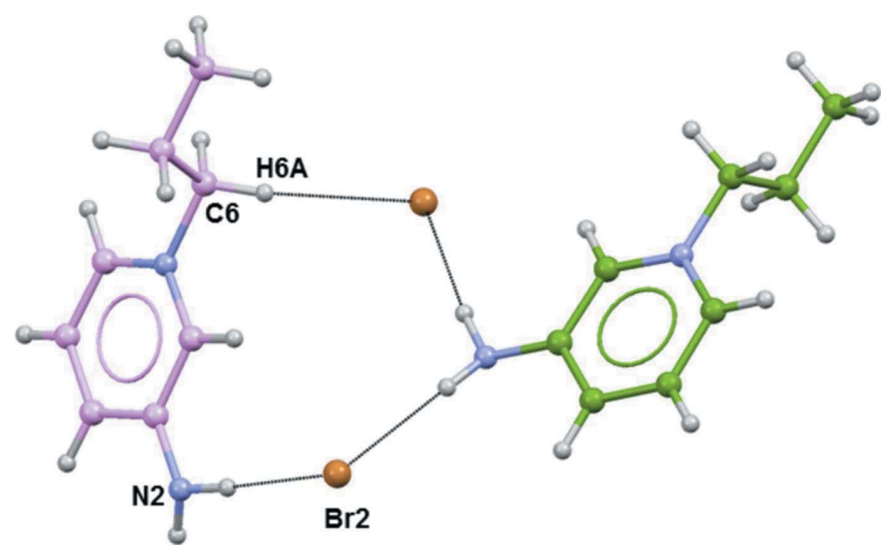

Figure 4

Part of the crystal structure of the title salt, showing the formation of an $R_{2}^{4}(12)$ ring motif (see Table 1 for details; only the interacting atoms are labelled).

\section{Database survey}

A search of the Cambridge Structural Database (Version 5.35, last update May 2014; Groom \& Allen, 2014) for 4-aminopyridinium halide salts gave nine hits, while a search for 3-aminopyridinium salts yielded eight hits. They all have different substituents at the pyridine ring $\mathrm{N}$ position, and include for example, 2-(3-aminopyridinium-1-yl)propanoate hydrobromide hemihydrate (CCDC refcode: IVAWUY; Kowalczyk et al., 2011), 2-(3-aminopyridinium-1-yl)-3-carboxypropanoate monohydrate (CCDC refcode: LAQGAN; Millán Corrales et al., 2012), 3-amino-1-(carboxymethyl)pyridinium chloride (CCDC refcode: PABTIX; Kowalczyk et al., 2010) and 3-Amino-1-(4-nitrobenzyl)pyridinium bromide (CCDC refcode: XEBFUG; Sundar et al., 2006). The mean planes of the substituent groups at the ring $\mathrm{N}$ atom make dihedral angles of $c a 80.3^{\circ}$ with the 3 -aminopyridinium ring in IVAWUY and $c a 86.6^{\circ}$ in PABTIX. In LAQGAN, the propanoate moiety is inclined at an angle of $c a 86.6^{\circ}$, and the

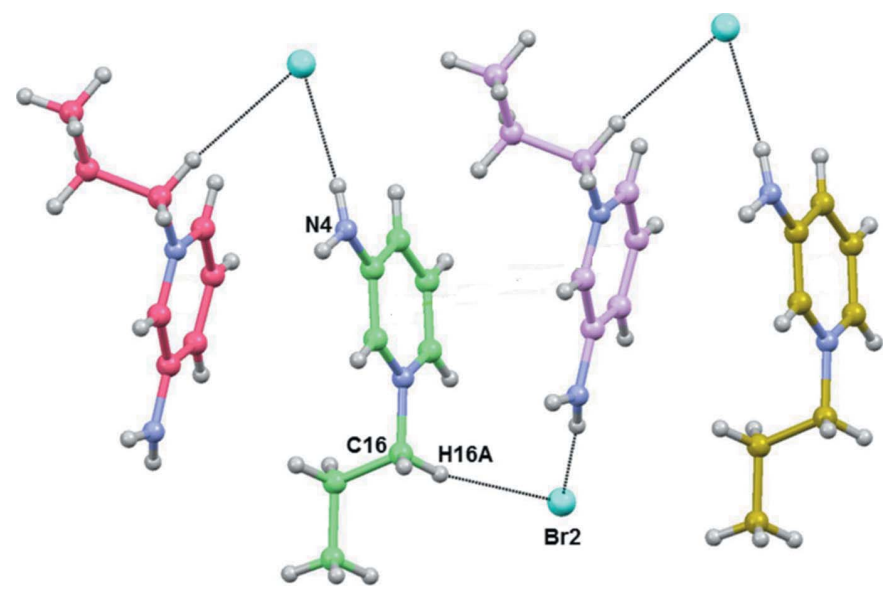

Figure 5

Part of the crystal structure of the title salt, showing the formation of a hydrogen-bonded chain that runs parallel to the $a$ axis (see Table 1 for details; only the interacting atoms are labelled).

Table 2

Experimental details.

Crystal data

Chemical formula

$M_{\mathrm{r}}$

Crystal system, space group

Temperature (K)

$a, b, c(\AA)$

$V\left(\AA^{3}\right)$

Z

Radiation type

$\mu\left(\mathrm{mm}^{-1}\right)$

Crystal size (mm)

Data collection

Diffractometer

Absorption correction

$T_{\min }, T_{\max }$

No. of measured, independent and observed $[I>2 \sigma(I)]$ reflections

$R_{\text {in }}$

$(\sin \theta / \lambda)_{\max }\left(\AA^{-1}\right)$

Refinement

$R\left[F^{2}>2 \sigma\left(F^{2}\right)\right], w R\left(F^{2}\right), S$

No. of reflections

No. of parameters

No. of restraints

$\mathrm{H}$-atom treatment

$\Delta \rho_{\max }, \Delta \rho_{\min }\left(\mathrm{e} \AA^{-3}\right)$

Computer programs: SMART and SAINT (Bruker, 2008), SHELXS2014 and SHELXL2014 (Sheldrick, 2008), PLATON (Spek, 2009), Mercury (Macrae et al., 2008) and PLATON (Spek, 2009).

carboxy moiety by $\mathrm{ca} 68.4^{\circ}$, with respect to the 3 -aminopyridinium ring. In XEBFUG, the 4-nitrobenzyl ring makes a dihedral angle of $c a 88.7^{\circ}$ with the 3-aminopyridinium ring.

\section{Synthesis and crystallization}

The title salt was prepared by dissolving 3-aminopyridine $(0.94 \mathrm{~g}, 10 \mathrm{mM})$ in dried acetone $(20 \mathrm{ml})$ and adding $n$-propyl bromide $(1.48 \mathrm{~g}, 12 \mathrm{mM})$. The reaction mixture was stirred at room temperature for $8 \mathrm{~h}$. The title salt precipitated as a white solid, which was filtered and washed with cold acetone and dried in vacuum to afford the stable salt. It was recrystallized from an aqueous ethanol solution giving colourless prismatic crystals.

\section{Refinement}

The details of crystal data, data collection and structure refinement are summarized in Table 2. The $\mathrm{N}$-bound $\mathrm{H}$ atoms were located in a difference Fourier map and freely refined. In the final cycles of refinement, the $\mathrm{H}$ atoms bound to atom $\mathrm{N} 2$ were refined with $U_{\text {iso }}(\mathrm{H})=1.1 U_{\text {eq }}(\mathrm{N})$. The C-bound $\mathrm{H}$ atoms were included in calculated positions and treated as riding atoms: $\mathrm{C}-\mathrm{H}=0.93-0.97 \AA$ with $U_{\text {iso }}(\mathrm{H})=1.5 U_{\text {eq }}(\mathrm{C})$ for methyl $\mathrm{H}$ atoms and $=1.2 U_{\mathrm{eq}}(\mathrm{C})$ for other $\mathrm{H}$ atoms. 


\section{Acknowledgements}

PV thanks the DST-FIST, New Delhi, for the NMR and XRD facilities at the School of Chemistry, Bharathidasan University, Tiruchirappalli, India. The authors thank Professor A. Ilangovan for his generous help. ST is extremely grateful to the management of SASTRA University for infrastructure and financial support (Professor TRR grant).

\section{References}

Anwar, N., Kosuge, H., Okada, S., Oikawa, H. \& Nakanishi, H. (2001). Jpn J. Appl. Phys. 40, 4213-4216.

Bruker (2008). APEX2, SAINT and SADABS. Bruker AXS Inc., Madison, Wisconsin, USA.

Calas, M., Ouattara, M., Piquet, G., Ziora, Z., Bordat, Y., Ancelin, M. L., Escale, R. \& Vial, H. (2007). J. Med. Chem. 50, 6307-6315.

Ezaki, S. \& Kokeguchi, N. (2006). Jpn Patent JP 2006171518.

Gama, Y., Suzuki, H. \& Narasaki, H. (1981). Jpn Patent JP 56139463.

Groom, C. R. \& Allen, F. H. (2014). Angew. Chem. Int. Ed. 53, 662671.

Ilangovan, A., Venkatesan, P., Sundararaman, M. \& Rajesh Kumar, R. (2012). Med. Chem. Res. 21, 694-702.
Kowalczyk, I., Katrusiak, A., Komasa, A. \& Szafran, M. (2011). J. Mol. Struct. 994, 13-20.

Kowalczyk, I., Katrusiak, A. \& Szafran, M. (2010). J. Mol. Struct. 979, 12-21.

Kupetis, G. K., Šaduikis, G., Nivinskien $\varnothing$, O. \& Eicher-Lorka, O. (2002). Monatsh. Chem. 133, 313-321.

Macrae, C. F., Bruno, I. J., Chisholm, J. A., Edgington, P. R., McCabe, P., Pidcock, E., Rodriguez-Monge, L., Taylor, R., van de Streek, J. \& Wood, P. A. (2008). J. Appl. Cryst. 41, 466-470.

Millán Corrales, G., Morales-Morales, D., Hernández-Ortega, S., Campos-Gaxiola, J. J. \& Cruz Enríquez, A. (2012). Acta Cryst. E68, o853.

Muldoon, M., Brennecke, J. F., Maginn, E. J., Scriven, E. F. V., McAteer, C. H. \& Murugan, R. (2010). US Patent 7687513, B1 20100330.

Petkovic, M., Seddon, K. R., Rebelo, L. P. \& Silva Pereira, C. (2011). Chem. Soc. Rev. 40, 1383-1403.

Sheldrick, G. M. (2008). Acta Cryst. A64, 112-122.

Spek, A. L. (2009). Acta Cryst. D65, 148-155.

Sundar, T. V., Parthasarathi, V., Sridhar, B., Venkatesan, P. \& Nallu, M. (2006). Acta Cryst. E62, o482-o484.

Sundararaman, M., Rajesh Kumar, R., Venkatesan, P. \& Ilangovan, A. (2013). J. Med. Microbiol. 62, 241-248.

Yamamoto, E., Yamaguchi, S. \& Nagamune, T. (2011). Appl. Biochem. Biotechnol. 164, 957-967.

Zhao, T. \& Sun, G. (2007). J. Appl. Polym. Sci. 103, 482-486. 


\section{supporting information}

Acta Cryst. (2014). E70, 580-583 [doi:10.1107/S1600536814025665]

\section{Crystal structure of 3-amino-1-propylpyridinium bromide}

\section{P. Venkatesan, V. Rajakannan and S. Thamotharan}

\section{Computing details}

Data collection: SMART (Bruker, 2008); cell refinement: SAINT (Bruker, 2008); data reduction: SAINT (Bruker, 2008); program(s) used to solve structure: SHELXS2014 (Sheldrick, 2008); program(s) used to refine structure: SHELXL2014 (Sheldrick, 2008); molecular graphics: PLATON (Spek, 2009) and Mercury (Macrae et al., 2008); software used to prepare material for publication: SHELXL2014 (Sheldrick, 2008) and PLATON (Spek, 2009).

\section{3-Amino-1-propylpyridinium bromide}

Crystal data

$\mathrm{C}_{8} \mathrm{H}_{13} \mathrm{~N}_{2}^{+} \cdot \mathrm{Br}^{-}$

$M_{r}=217.11$

Orthorhombic, $\mathrm{Pbca}$

$a=8.2937(1) \AA$

$b=17.4137(3) \AA$

$c=26.9626(4) \AA$

$V=3894.05(10) \AA^{3}$

$Z=16$

$F(000)=1760$

\section{Data collection}

Bruker SMART CCD area-detector diffractometer

Radiation source: sealed tube

Graphite monochromator

phi and $\omega$ scans

Absorption correction: multi-scan

(SADABS; Bruker, 2008)

$T_{\min }=0.635, T_{\max }=0.681$

Refinement

Refinement on $F^{2}$

Least-squares matrix: full

$R\left[F^{2}>2 \sigma\left(F^{2}\right)\right]=0.036$

$w R\left(F^{2}\right)=0.085$

$S=1.00$

4491 reflections

214 parameters

4 restraints

Hydrogen site location: mixed
$D_{\mathrm{x}}=1.481 \mathrm{Mg} \mathrm{m}^{-3}$

Mo $K \alpha$ radiation, $\lambda=0.71073 \AA$

Cell parameters from 5144 reflections

$\theta=2.8-23.4^{\circ}$

$\mu=4.17 \mathrm{~mm}^{-1}$

$T=296 \mathrm{~K}$

Prismolourec, colourless

$0.12 \times 0.10 \times 0.10 \mathrm{~mm}$

21922 measured reflections

4491 independent reflections

2698 reflections with $I>2 \sigma(I)$

$R_{\text {int }}=0.043$

$\theta_{\max }=27.6^{\circ}, \theta_{\min }=2.8^{\circ}$

$h=-10 \rightarrow 10$

$k=-22 \rightarrow 22$

$l=-35 \rightarrow 30$

$\mathrm{H}$ atoms treated by a mixture of independent and constrained refinement

$w=1 /\left[\sigma^{2}\left(F_{0}^{2}\right)+(0.0353 P)^{2}+1.288 P\right]$

where $P=\left(F_{\mathrm{o}}{ }^{2}+2 F_{\mathrm{c}}{ }^{2}\right) / 3$

$(\Delta / \sigma)_{\max }<0.001$

$\Delta \rho_{\max }=0.44$ e $\AA^{-3}$

$\Delta \rho_{\min }=-0.31$ e $\AA^{-3}$

Extinction correction: SHELXL2014 (Sheldrick, 2008), $\mathrm{Fc}^{*}=\mathrm{kFc}\left[1+0.001 \mathrm{xFc}^{2} \lambda^{3} / \sin (2 \theta)\right]^{-1 / 4}$

Extinction coefficient: 0.00312 (18) 


\section{Special details}

Experimental. The minimum and maximum absorption values stated above are those calculated in SHELXL2014/6 from the given crystal dimensions. The ratio of minimum to maximum apparent transmission was determined experimentally as 0.639091 .

Geometry. All e.s.d.'s (except the e.s.d. in the dihedral angle between two 1.s. planes) are estimated using the full covariance matrix. The cell e.s.d.'s are taken into account individually in the estimation of e.s.d.'s in distances, angles and torsion angles; correlations between e.s.d.'s in cell parameters are only used when they are defined by crystal symmetry. An approximate (isotropic) treatment of cell e.s.d.'s is used for estimating e.s.d.'s involving 1.s. planes.

Fractional atomic coordinates and isotropic or equivalent isotropic displacement parameters $\left(\AA^{2}\right)$

\begin{tabular}{|c|c|c|c|c|}
\hline & $x$ & $y$ & $z$ & $U_{\text {iso }} * / U_{\text {eq }}$ \\
\hline Br1 & $0.82809(4)$ & 0.05269 (2) & 0.70989 (2) & 0.06468 (14) \\
\hline N1 & -0.0380 & $0.39600(15)$ & $0.40238(10)$ & $0.0563(7)$ \\
\hline $\mathrm{N} 2$ & $-0.3901(4)$ & $0.3230(2)$ & $0.46352(12)$ & $0.0885(10)$ \\
\hline $\mathrm{H} 2 \mathrm{~A}$ & $-0.472(3)$ & $0.2941(19)$ & $0.4526(13)$ & $0.097 *$ \\
\hline $\mathrm{H} 2 \mathrm{~B}$ & $-0.341(4)$ & $0.305(2)$ & $0.4909(10)$ & $0.097^{*}$ \\
\hline $\mathrm{C} 1$ & $-0.1328(4)$ & $0.36563(18)$ & $0.43751(12)$ & $0.0566(8)$ \\
\hline $\mathrm{H} 1$ & -0.0897 & 0.3536 & 0.4684 & $0.068^{*}$ \\
\hline $\mathrm{C} 4$ & $-0.2546(4)$ & 0.4017 (2) & 0.34649 (13) & $0.0644(9)$ \\
\hline H4 & -0.2950 & 0.4143 & 0.3154 & $0.077^{*}$ \\
\hline $\mathrm{C} 2$ & $-0.2949(4)$ & $0.35190(19)$ & $0.42823(12)$ & $0.0556(8)$ \\
\hline $\mathrm{C} 7$ & $0.1580(4)$ & $0.4864(2)$ & $0.43960(13)$ & $0.0626(9)$ \\
\hline H7A & 0.0959 & 0.4875 & 0.4701 & $0.075^{*}$ \\
\hline H7B & 0.1185 & 0.5272 & 0.4183 & $0.075^{*}$ \\
\hline $\mathrm{C} 3$ & -0.3540 & $0.37094(18)$ & $0.38170(12)$ & $0.0601(9)$ \\
\hline $\mathrm{H} 3$ & -0.4622 & 0.3627 & 0.3744 & $0.072 *$ \\
\hline $\mathrm{C} 8$ & $0.3336(4)$ & $0.5006(2)$ & $0.45141(14)$ & $0.0739(10)$ \\
\hline H8A & 0.3448 & 0.5494 & 0.4675 & $0.111^{*}$ \\
\hline H8B & 0.3724 & 0.4608 & 0.4730 & $0.111 *$ \\
\hline $\mathrm{H} 8 \mathrm{C}$ & 0.3951 & 0.5005 & 0.4212 & $0.111^{*}$ \\
\hline C6 & $0.1340(4)$ & $0.4116(2)$ & $0.41464(13)$ & $0.0653(9)$ \\
\hline H6A & 0.1739 & 0.3711 & 0.4360 & $0.078 *$ \\
\hline H6B & 0.1969 & 0.4108 & 0.3843 & $0.078^{*}$ \\
\hline $\mathrm{C} 5$ & $-0.0945(5)$ & $0.4141(2)$ & $0.35731(13)$ & $0.0658(9)$ \\
\hline H5 & -0.0260 & 0.4348 & 0.3335 & $0.079 *$ \\
\hline $\mathrm{Br} 2$ & $0.29379(4)$ & $0.21682(2)$ & $0.42595(2)$ & $0.06172(14)$ \\
\hline N3 & $0.9614(3)$ & $0.15602(14)$ & $0.30646(8)$ & $0.0478(6)$ \\
\hline N4 & 0.8705 (4) & $0.1118(2)$ & $0.17923(11)$ & $0.0678(8)$ \\
\hline $\mathrm{H} 4 \mathrm{~A}$ & $0.815(3)$ & $0.0725(14)$ & $0.1844(13)$ & $0.064(11)^{*}$ \\
\hline H4B & 0.859 (4) & $0.1350(18)$ & $0.1507(8)$ & $0.072(11)^{*}$ \\
\hline $\mathrm{C} 11$ & $0.9112(3)$ & $0.11840(17)$ & $0.26620(10)$ & $0.0455(7)$ \\
\hline H11 & 0.8716 & 0.0687 & 0.2694 & $0.055^{*}$ \\
\hline C16 & 0.9419 (4) & 0.1197 (2) & $0.35593(10)$ & $0.0558(8)$ \\
\hline H16A & 1.0258 & 0.1379 & 0.3780 & $0.067^{*}$ \\
\hline H16B & 0.9528 & 0.0644 & 0.3528 & $0.067^{*}$ \\
\hline $\mathrm{C} 13$ & $0.9756(4)$ & $0.22690(19)$ & $0.21730(12)$ & $0.0612(9)$ \\
\hline H13 & 0.9786 & 0.2522 & 0.1869 & $0.073^{*}$ \\
\hline
\end{tabular}


supporting information

$\begin{array}{lllll}\text { C15 } & 1.0220(4) & 0.22732(19) & 0.30409(13) & 0.0605(9) \\ \text { H15 } & 1.0591 & 0.2517 & 0.3326 & 0.073^{*} \\ \text { C12 } & 0.9171(3) & 0.15187(18) & 0.21987(10) & 0.0477(7) \\ \text { C14 } & 1.0285(4) & 0.26351(19) & 0.25917(14) & 0.0679(9) \\ \text { H14 } & 1.0690 & 0.3132 & 0.2570 & 0.081^{*} \\ \text { C18 } & 0.7575(5) & 0.1097(3) & 0.42914(14) & 0.1018(15) \\ \text { H18A } & 0.6522 & 0.1233 & 0.4411 & 0.153^{*} \\ \text { H18B } & 0.7691 & 0.0549 & 0.4294 & 0.153^{*} \\ \text { H18C } & 0.8379 & 0.1323 & 0.4502 & 0.153^{*} \\ \text { C17 } & 0.7780(4) & 0.1388(3) & 0.37761(13) & 0.0812(12) \\ \text { H17A } & 0.7637 & 0.1940 & 0.3775 & 0.097^{*} \\ \text { H17B } & 0.6949 & 0.1166 & 0.3567 & 0.097^{*}\end{array}$

Atomic displacement parameters $\left(\AA^{2}\right)$

\begin{tabular}{|c|c|c|c|c|c|c|}
\hline & $U^{11}$ & $U^{22}$ & $U^{33}$ & $U^{12}$ & $U^{13}$ & $U^{23}$ \\
\hline Br1 & $0.0623(2)$ & $0.0678(3)$ & $0.0640(2)$ & $-0.01030(16)$ & $0.00528(16)$ & $-0.00851(18)$ \\
\hline N1 & $0.0529(16)$ & $0.0501(16)$ & $0.0659(18)$ & $-0.0015(13)$ & $0.0095(14)$ & $-0.0127(14)$ \\
\hline $\mathrm{N} 2$ & $0.083(2)$ & $0.111(3)$ & $0.072(2)$ & $-0.037(2)$ & $-0.0128(18)$ & $0.011(2)$ \\
\hline $\mathrm{C} 1$ & $0.061(2)$ & $0.053(2)$ & $0.056(2)$ & $-0.0037(16)$ & $-0.0051(16)$ & $-0.0058(16)$ \\
\hline $\mathrm{C} 4$ & $0.070(2)$ & $0.061(2)$ & $0.062(2)$ & $-0.0034(19)$ & $0.0026(19)$ & $-0.0106(18)$ \\
\hline $\mathrm{C} 2$ & $0.056(2)$ & $0.053(2)$ & $0.058(2)$ & $-0.0156(15)$ & $0.0041(16)$ & $-0.0062(17)$ \\
\hline $\mathrm{C} 7$ & $0.057(2)$ & $0.063(2)$ & $0.068(2)$ & $0.0004(17)$ & $0.0003(16)$ & $-0.0092(19)$ \\
\hline $\mathrm{C} 3$ & $0.056(2)$ & $0.061(2)$ & $0.063(2)$ & $-0.0030(17)$ & $-0.0124(17)$ & -0.0115 (18) \\
\hline $\mathrm{C} 8$ & $0.059(2)$ & $0.079(3)$ & $0.084(3)$ & $-0.0115(19)$ & $-0.0043(18)$ & $0.002(2)$ \\
\hline C6 & $0.0474(19)$ & $0.064(2)$ & $0.085(2)$ & $-0.0005(17)$ & $0.0056(17)$ & $-0.008(2)$ \\
\hline $\mathrm{C} 5$ & $0.080(3)$ & $0.060(2)$ & $0.057(2)$ & -0.0028 (19) & $0.0154(19)$ & $-0.0059(18)$ \\
\hline $\mathrm{Br} 2$ & $0.0741(3)$ & $0.0604(2)$ & $0.0506(2)$ & $-0.01163(16)$ & $0.00011(15)$ & $-0.00427(16)$ \\
\hline N3 & $0.0482(14)$ & $0.0465(16)$ & $0.0486(15)$ & $0.0023(12)$ & $-0.0029(11)$ & $0.0027(12)$ \\
\hline N4 & $0.087(2)$ & $0.072(2)$ & $0.0449(18)$ & $-0.0176(18)$ & $-0.0075(16)$ & $0.0096(17)$ \\
\hline $\mathrm{C} 11$ & $0.0466(17)$ & $0.0398(17)$ & $0.0500(17)$ & $-0.0013(13)$ & $-0.0030(14)$ & $0.0008(15)$ \\
\hline $\mathrm{C} 16$ & $0.057(2)$ & $0.063(2)$ & $0.0474(17)$ & $-0.0012(16)$ & $-0.0069(15)$ & $0.0026(16)$ \\
\hline $\mathrm{C} 13$ & $0.069(2)$ & $0.056(2)$ & $0.058(2)$ & $-0.0019(17)$ & $0.0035(17)$ & $0.0141(18)$ \\
\hline $\mathrm{C} 15$ & $0.068(2)$ & $0.049(2)$ & $0.064(2)$ & $-0.0054(17)$ & $-0.0065(17)$ & $-0.0074(17)$ \\
\hline $\mathrm{C} 12$ & $0.0483(17)$ & $0.0518(19)$ & $0.0430(18)$ & $-0.0003(14)$ & $-0.0023(14)$ & $0.0074(15)$ \\
\hline $\mathrm{C} 14$ & $0.079(2)$ & $0.045(2)$ & $0.080(3)$ & $-0.0076(17)$ & $-0.001(2)$ & $0.0045(19)$ \\
\hline $\mathrm{C} 18$ & $0.086(3)$ & $0.141(4)$ & $0.078(3)$ & $0.011(3)$ & $0.015(2)$ & $0.017(3)$ \\
\hline C17 & $0.066(2)$ & $0.115(3)$ & $0.063(2)$ & $0.003(2)$ & $0.0002(18)$ & $0.022(2)$ \\
\hline
\end{tabular}

Geometric parameters $\left(\AA,{ }^{\circ}\right)$

\begin{tabular}{llll}
\hline $\mathrm{N} 1-\mathrm{C} 5$ & $1.340(4)$ & $\mathrm{N} 3-\mathrm{C} 11$ & $1.334(3)$ \\
$\mathrm{N} 1-\mathrm{C} 1$ & $1.340(4)$ & $\mathrm{N} 3-\mathrm{C} 15$ & $1.341(4)$ \\
$\mathrm{N} 1-\mathrm{C} 6$ & $1.489(4)$ & $\mathrm{N} 3-\mathrm{C} 16$ & $1.485(3)$ \\
$\mathrm{N} 2-\mathrm{C} 2$ & $1.336(4)$ & $\mathrm{N} 4-\mathrm{C} 12$ & $1.356(4)$ \\
$\mathrm{N} 2-\mathrm{H} 2 \mathrm{~A}$ & $0.896(18)$ & $\mathrm{N} 4-\mathrm{H} 4 \mathrm{~A}$ & $0.834(18)$ \\
$\mathrm{N} 2-\mathrm{H} 2 \mathrm{~B}$ & $0.897(18)$ & $\mathrm{N} 4-\mathrm{H} 4 \mathrm{~B}$ & $0.874(18)$ \\
$\mathrm{C} 1-\mathrm{C} 2$ & $1.388(4)$ & $\mathrm{C} 11-\mathrm{C} 12$ & $1.379(4)$
\end{tabular}




\begin{tabular}{|c|c|c|c|}
\hline $\mathrm{C} 1-\mathrm{H} 1$ & 0.9300 & $\mathrm{C} 11-\mathrm{H} 11$ & 0.9300 \\
\hline $\mathrm{C} 4-\mathrm{C} 3$ & $1.366(4)$ & $\mathrm{C} 16-\mathrm{C} 17$ & $1.517(4)$ \\
\hline $\mathrm{C} 4-\mathrm{C} 5$ & $1.377(5)$ & $\mathrm{C} 16-\mathrm{H} 16 \mathrm{~A}$ & 0.9700 \\
\hline $\mathrm{C} 4-\mathrm{H} 4$ & 0.9300 & $\mathrm{C} 16-\mathrm{H} 16 \mathrm{~B}$ & 0.9700 \\
\hline $\mathrm{C} 2-\mathrm{C} 3$ & $1.387(4)$ & $\mathrm{C} 13-\mathrm{C} 14$ & $1.369(4)$ \\
\hline $\mathrm{C} 7-\mathrm{C} 6$ & $1.479(4)$ & $\mathrm{C} 13-\mathrm{C} 12$ & $1.395(4)$ \\
\hline $\mathrm{C} 7-\mathrm{C} 8$ & $1.511(4)$ & $\mathrm{C} 13-\mathrm{H} 13$ & 0.9300 \\
\hline C7-H7A & 0.9700 & $\mathrm{C} 15-\mathrm{C} 14$ & $1.366(4)$ \\
\hline $\mathrm{C} 7-\mathrm{H} 7 \mathrm{~B}$ & 0.9700 & $\mathrm{C} 15-\mathrm{H} 15$ & 0.9300 \\
\hline $\mathrm{C} 3-\mathrm{H} 3$ & 0.9300 & $\mathrm{C} 14-\mathrm{H} 14$ & 0.9300 \\
\hline $\mathrm{C} 8-\mathrm{H} 8 \mathrm{~A}$ & 0.9600 & $\mathrm{C} 18-\mathrm{C} 17$ & $1.488(5)$ \\
\hline $\mathrm{C} 8-\mathrm{H} 8 \mathrm{~B}$ & 0.9600 & $\mathrm{C} 18-\mathrm{H} 18 \mathrm{~A}$ & 0.9600 \\
\hline $\mathrm{C} 8-\mathrm{H} 8 \mathrm{C}$ & 0.9600 & $\mathrm{C} 18-\mathrm{H} 18 \mathrm{~B}$ & 0.9600 \\
\hline C6-H6A & 0.9700 & $\mathrm{C} 18-\mathrm{H} 18 \mathrm{C}$ & 0.9600 \\
\hline C6- $-\mathrm{H} 6 \mathrm{~B}$ & 0.9700 & C17-H17A & 0.9700 \\
\hline $\mathrm{C} 5-\mathrm{H} 5$ & 0.9300 & C17-H17B & 0.9700 \\
\hline $\mathrm{C} 5-\mathrm{N} 1-\mathrm{C} 1$ & $121.9(3)$ & $\mathrm{C} 11-\mathrm{N} 3-\mathrm{C} 15$ & $122.2(3)$ \\
\hline $\mathrm{C} 5-\mathrm{N} 1-\mathrm{C} 6$ & $119.6(3)$ & $\mathrm{C} 11-\mathrm{N} 3-\mathrm{C} 16$ & $119.2(3)$ \\
\hline $\mathrm{C} 1-\mathrm{N} 1-\mathrm{C} 6$ & $118.5(3)$ & $\mathrm{C} 15-\mathrm{N} 3-\mathrm{C} 16$ & $118.6(3)$ \\
\hline $\mathrm{C} 2-\mathrm{N} 2-\mathrm{H} 2 \mathrm{~A}$ & $115(3)$ & $\mathrm{C} 12-\mathrm{N} 4-\mathrm{H} 4 \mathrm{~A}$ & $116(2)$ \\
\hline $\mathrm{C} 2-\mathrm{N} 2-\mathrm{H} 2 \mathrm{~B}$ & $117(2)$ & $\mathrm{C} 12-\mathrm{N} 4-\mathrm{H} 4 \mathrm{~B}$ & $120(2)$ \\
\hline $\mathrm{H} 2 \mathrm{~A}-\mathrm{N} 2-\mathrm{H} 2 \mathrm{~B}$ & $115(4)$ & $\mathrm{H} 4 \mathrm{~A}-\mathrm{N} 4-\mathrm{H} 4 \mathrm{~B}$ & $118(3)$ \\
\hline $\mathrm{N} 1-\mathrm{C} 1-\mathrm{C} 2$ & $120.6(3)$ & $\mathrm{N} 3-\mathrm{C} 11-\mathrm{C} 12$ & $121.2(3)$ \\
\hline $\mathrm{N} 1-\mathrm{C} 1-\mathrm{H} 1$ & 119.7 & $\mathrm{~N} 3-\mathrm{C} 11-\mathrm{H} 11$ & 119.4 \\
\hline $\mathrm{C} 2-\mathrm{C} 1-\mathrm{H} 1$ & 119.7 & $\mathrm{C} 12-\mathrm{C} 11-\mathrm{H} 11$ & 119.4 \\
\hline $\mathrm{C} 3-\mathrm{C} 4-\mathrm{C} 5$ & $119.7(3)$ & $\mathrm{N} 3-\mathrm{C} 16-\mathrm{C} 17$ & $110.5(2)$ \\
\hline $\mathrm{C} 3-\mathrm{C} 4-\mathrm{H} 4$ & 120.1 & $\mathrm{~N} 3-\mathrm{C} 16-\mathrm{H} 16 \mathrm{~A}$ & 109.6 \\
\hline $\mathrm{C} 5-\mathrm{C} 4-\mathrm{H} 4$ & 120.1 & $\mathrm{C} 17-\mathrm{C} 16-\mathrm{H} 16 \mathrm{~A}$ & 109.6 \\
\hline $\mathrm{N} 2-\mathrm{C} 2-\mathrm{C} 3$ & $121.7(3)$ & $\mathrm{N} 3-\mathrm{C} 16-\mathrm{H} 16 \mathrm{~B}$ & 109.6 \\
\hline $\mathrm{N} 2-\mathrm{C} 2-\mathrm{C} 1$ & $120.6(3)$ & $\mathrm{C} 17-\mathrm{C} 16-\mathrm{H} 16 \mathrm{~B}$ & 109.6 \\
\hline $\mathrm{C} 3-\mathrm{C} 2-\mathrm{C} 1$ & $117.7(3)$ & $\mathrm{H} 16 \mathrm{~A}-\mathrm{C} 16-\mathrm{H} 16 \mathrm{~B}$ & 108.1 \\
\hline $\mathrm{C} 6-\mathrm{C} 7-\mathrm{C} 8$ & $111.6(3)$ & $\mathrm{C} 14-\mathrm{C} 13-\mathrm{C} 12$ & $120.4(3)$ \\
\hline $\mathrm{C} 6-\mathrm{C} 7-\mathrm{H} 7 \mathrm{~A}$ & 109.3 & $\mathrm{C} 14-\mathrm{C} 13-\mathrm{H} 13$ & 119.8 \\
\hline $\mathrm{C} 8-\mathrm{C} 7-\mathrm{H} 7 \mathrm{~A}$ & 109.3 & $\mathrm{C} 12-\mathrm{C} 13-\mathrm{H} 13$ & 119.8 \\
\hline $\mathrm{C} 6-\mathrm{C} 7-\mathrm{H} 7 \mathrm{~B}$ & 109.3 & $\mathrm{~N} 3-\mathrm{C} 15-\mathrm{C} 14$ & $119.0(3)$ \\
\hline $\mathrm{C} 8-\mathrm{C} 7-\mathrm{H} 7 \mathrm{~B}$ & 109.3 & $\mathrm{~N} 3-\mathrm{C} 15-\mathrm{H} 15$ & 120.5 \\
\hline $\mathrm{H} 7 \mathrm{~A}-\mathrm{C} 7-\mathrm{H} 7 \mathrm{~B}$ & 108.0 & $\mathrm{C} 14-\mathrm{C} 15-\mathrm{H} 15$ & 120.5 \\
\hline $\mathrm{C} 4-\mathrm{C} 3-\mathrm{C} 2$ & $120.6(3)$ & $\mathrm{N} 4-\mathrm{C} 12-\mathrm{C} 11$ & $120.3(3)$ \\
\hline $\mathrm{C} 4-\mathrm{C} 3-\mathrm{H} 3$ & 119.7 & $\mathrm{~N} 4-\mathrm{C} 12-\mathrm{C} 13$ & $122.8(3)$ \\
\hline $\mathrm{C} 2-\mathrm{C} 3-\mathrm{H} 3$ & 119.7 & $\mathrm{C} 11-\mathrm{C} 12-\mathrm{C} 13$ & $116.9(3)$ \\
\hline $\mathrm{C} 7-\mathrm{C} 8-\mathrm{H} 8 \mathrm{~A}$ & 109.5 & $\mathrm{C} 15-\mathrm{C} 14-\mathrm{C} 13$ & $120.2(3)$ \\
\hline $\mathrm{C} 7-\mathrm{C} 8-\mathrm{H} 8 \mathrm{~B}$ & 109.5 & $\mathrm{C} 15-\mathrm{C} 14-\mathrm{H} 14$ & 119.9 \\
\hline $\mathrm{H} 8 \mathrm{~A}-\mathrm{C} 8-\mathrm{H} 8 \mathrm{~B}$ & 109.5 & $\mathrm{C} 13-\mathrm{C} 14-\mathrm{H} 14$ & 119.9 \\
\hline $\mathrm{C} 7-\mathrm{C} 8-\mathrm{H} 8 \mathrm{C}$ & 109.5 & $\mathrm{C} 17-\mathrm{C} 18-\mathrm{H} 18 \mathrm{~A}$ & 109.5 \\
\hline $\mathrm{H} 8 \mathrm{~A}-\mathrm{C} 8-\mathrm{H} 8 \mathrm{C}$ & 109.5 & $\mathrm{C} 17-\mathrm{C} 18-\mathrm{H} 18 \mathrm{~B}$ & 109.5 \\
\hline $\mathrm{H} 8 \mathrm{~B}-\mathrm{C} 8-\mathrm{H} 8 \mathrm{C}$ & 109.5 & $\mathrm{H} 18 \mathrm{~A}-\mathrm{C} 18-\mathrm{H} 18 \mathrm{~B}$ & 109.5 \\
\hline $\mathrm{C} 7-\mathrm{C} 6-\mathrm{N} 1$ & $113.0(3)$ & $\mathrm{C} 17-\mathrm{C} 18-\mathrm{H} 18 \mathrm{C}$ & 109.5 \\
\hline
\end{tabular}




$\begin{array}{llll}\mathrm{C} 7-\mathrm{C} 6-\mathrm{H} 6 \mathrm{~A} & 109.0 & \mathrm{H} 18 \mathrm{~A}-\mathrm{C} 18-\mathrm{H} 18 \mathrm{C} & 109.5 \\ \mathrm{~N} 1-\mathrm{C} 6-\mathrm{H} 6 \mathrm{~A} & 109.0 & \mathrm{H} 18 \mathrm{~B}-\mathrm{C} 18-\mathrm{H} 18 \mathrm{C} & 109.5 \\ \mathrm{C} 7-\mathrm{C} 6-\mathrm{H} 6 \mathrm{~B} & 109.0 & \mathrm{C} 18-\mathrm{C} 17-\mathrm{C} 16 & 112.8(3) \\ \mathrm{N} 1-\mathrm{C} 6-\mathrm{H} 6 \mathrm{~B} & 109.0 & \mathrm{C} 18-\mathrm{C} 17-\mathrm{H} 17 \mathrm{~A} & 109.0 \\ \mathrm{H} 6 \mathrm{~A}-\mathrm{C} 6-\mathrm{H} 6 \mathrm{~B} & 107.8 & \mathrm{C} 16-\mathrm{C} 17-\mathrm{H} 17 \mathrm{~A} & 109.0 \\ \mathrm{~N} 1-\mathrm{C} 5-\mathrm{C} 4 & 119.6(3) & \mathrm{C} 18-\mathrm{C} 17-\mathrm{H} 17 \mathrm{~B} & 109.0 \\ \mathrm{~N} 1-\mathrm{C} 5-\mathrm{H} 5 & 120.2 & \mathrm{C} 16-\mathrm{C} 17-\mathrm{H} 17 \mathrm{~B} & 109.0 \\ \mathrm{C} 4-\mathrm{C} 5-\mathrm{H} 5 & 120.2 & \mathrm{H} 17 \mathrm{~A}-\mathrm{C} 17-\mathrm{H} 17 \mathrm{~B} & 107.8 \\ & & & \\ \mathrm{C} 5-\mathrm{N} 1-\mathrm{C} 1-\mathrm{C} 2 & -0.1(5) & \mathrm{C} 15-\mathrm{N} 3-\mathrm{C} 11-\mathrm{C} 12 & -1.1(4) \\ \mathrm{C} 6-\mathrm{N} 1-\mathrm{C} 1-\mathrm{C} 2 & 178.3(3) & \mathrm{C} 16-\mathrm{N} 3-\mathrm{C} 11-\mathrm{C} 12 & 175.7(3) \\ \mathrm{N} 1-\mathrm{C} 1-\mathrm{C} 2-\mathrm{N} 2 & -178.7(3) & \mathrm{C} 11-\mathrm{N} 3-\mathrm{C} 16-\mathrm{C} 17 & -88.0(3) \\ \mathrm{N} 1-\mathrm{C} 1-\mathrm{C} 2-\mathrm{C} 3 & -0.2(5) & \mathrm{C} 15-\mathrm{N} 3-\mathrm{C} 16-\mathrm{C} 17 & 88.9(3) \\ \mathrm{C} 5-\mathrm{C} 4-\mathrm{C} 3-\mathrm{C} 2 & -0.1(5) & \mathrm{C} 11-\mathrm{N} 3-\mathrm{C} 15-\mathrm{C} 14 & 1.9(5) \\ \mathrm{N} 2-\mathrm{C} 2-\mathrm{C} 3-\mathrm{C} 4 & 178.8(3) & \mathrm{C} 16-\mathrm{N} 3-\mathrm{C} 15-\mathrm{C} 14 & -174.9(3) \\ \mathrm{C} 1-\mathrm{C} 2-\mathrm{C} 3-\mathrm{C} 4 & 0.3(5) & \mathrm{N} 3-\mathrm{C} 11-\mathrm{C} 12-\mathrm{N} 4 & 177.9(3) \\ \mathrm{C} 8-\mathrm{C} 7-\mathrm{C} 6-\mathrm{N} 1 & -179.9(3) & \mathrm{N} 3-\mathrm{C} 11-\mathrm{C} 12-\mathrm{C} 13 & -0.9(4) \\ \mathrm{C} 5-\mathrm{N} 1-\mathrm{C} 6-\mathrm{C} 7 & 94.3(4) & \mathrm{C} 14-\mathrm{C} 13-\mathrm{C} 12-\mathrm{N} 4 & -176.8(3) \\ \mathrm{C} 1-\mathrm{N} 1-\mathrm{C} 6-\mathrm{C} 7 & -84.2(4) & \mathrm{C} 14-\mathrm{C} 13-\mathrm{C} 12-\mathrm{C} 11 & 1.9(4) \\ \mathrm{C} 1-\mathrm{N} 1-\mathrm{C} 5-\mathrm{C} 4 & 0.4(5) & \mathrm{N} 3-\mathrm{C} 15-\mathrm{C} 14-\mathrm{C} 13 & -0.8(5) \\ \mathrm{C} 6-\mathrm{N} 1-\mathrm{C} 5-\mathrm{C} 4 & -178.0(3) & \mathrm{C} 12-\mathrm{C} 13-\mathrm{C} 14-\mathrm{C} 15 & -1.1(5) \\ \mathrm{C} 3-\mathrm{C} 4-\mathrm{C} 5-\mathrm{N} 1 & -0.3(5) & \mathrm{N} 3-\mathrm{C} 16-\mathrm{C} 17-\mathrm{C} 18 & -174.8(3)\end{array}$

Hydrogen-bond geometry $\left(\AA,{ }^{\circ}\right)$

\begin{tabular}{lllll}
\hline$D-\mathrm{H} \cdots A$ & $D-\mathrm{H}$ & $\mathrm{H} \cdots A$ & $D \cdots A$ & $D-\mathrm{H} \cdots A$ \\
\hline $\mathrm{N} 2-\mathrm{H} 2 A \cdots \mathrm{Br} 2^{\mathrm{i}}$ & $0.90(2)$ & $2.47(2)$ & $3.364(3)$ & $177(4)$ \\
$\mathrm{N} 2-\mathrm{H} 2 B \cdots \mathrm{Br} 2^{\mathrm{ii}}$ & $0.90(2)$ & $2.54(2)$ & $3.419(3)$ & $168(4)$ \\
$\mathrm{N} 4-\mathrm{H} 4 A \cdots \mathrm{Br} 1^{\mathrm{iii}}$ & $0.83(2)$ & $2.58(2)$ & $3.406(3)$ & $172(3)$ \\
$\mathrm{N} 4-\mathrm{H} 4 B \cdots \mathrm{Br} 2^{\mathrm{iv}}$ & $0.87(2)$ & $2.57(2)$ & $3.434(3)$ & $171(3)$ \\
$\mathrm{C} 6-\mathrm{H} 6 A \cdots \mathrm{Br} 2$ & 0.97 & 2.88 & $3.655(4)$ & 138 \\
$\mathrm{C} 6-\mathrm{H} 6 B \cdots \mathrm{Br} 1^{\mathrm{ii}}$ & 0.97 & 2.84 & $3.775(4)$ & 163 \\
$\mathrm{C} 16-\mathrm{H} 16 A \cdots \mathrm{Br} 2^{\mathrm{v}}$ & 0.97 & 2.91 & $3.866(3)$ & 167 \\
\hline
\end{tabular}

Symmetry codes: (i) $x-1, y, z$; (ii) $x-1 / 2,-y+1 / 2,-z+1$; (iii) $-x+3 / 2,-y, z-1 / 2$; (iv) $x+1 / 2, y,-z+1 / 2$; (v) $x+1, y, z$. 